\title{
Factores asociados con el uso de tecnologías en información y comunicación en residentes de medicina familiar.
}

\section{Factors associated with the use of information and communication technologies in family medicine residents.}

\author{
Esaú Rojas Carrillo ${ }^{1 *}$, Veronica Benites Godinez ${ }^{2}$, Luisa Elizabeth Velasco \\ González ${ }^{3}$, Adriana Gabriela Ramírez Corona ${ }^{4}$, Jesús Adrián López Morán ${ }^{5}$, Iris \\ Jocelyn Parrao Alcántara ${ }^{6}$, Eduardo Manuel Benítez Godínez ${ }^{7}$
}

\footnotetext{
1 Unidad de Medicina Familiar No. 24 "Ignacio García Tellez", Instituto Mexicano del Seguro social. Tepic, Nayarit, México; dr esau@hotmail.com.mx, ORCID ID https:/ / orcid.org/0000-0003-2729-5497

2 Órgano de Operación Administrativa Desconcentrada Estatal de Nayarit, Instituto Mexicano del Seguro Social. Tepic, Nayarit, México. Unidad Académica de Medicina , Universidad Autónoma de Nayarit ORCID ID https:/ / orcid.org/0000-0002-7678-5006

${ }^{3}$ Unidad de Medicina Familiar No. 24 "Ignacio García Tellez”, Instituto Mexicano del Seguro social. Tepic, Nayarit, México. ORCID ID https:/ / orcid.org/0000-0002-3792-1538

${ }^{4}$ Unidad de Medicina Familiar No. 24 “Ignacio García Tellez", Instituto Mexicano del Seguro social. Tepic, Nayarit, México. ORCID ID https:/ / orcid.org/0000-0002-0569-7543

${ }^{5}$ Hospital General de Zona con Medicina Familiar No. 10, Instituto Mexicano del Seguro Social, Santiago Ixcuintla, Nayarit, México. https:/ / orcid.org/0000-0002-9857-5114

${ }^{6}$ Hospital General de Zona 33. Instituto Mexicano del Seguro Social, Bahía de Banderas Nayarit, México ORCID ID https:/ / orcid.org/0000-0002-2651-6908

${ }^{7}$ Instituto Universitario Modelo de Nayarit, Tepic Nayarit México ORCID ID https:/ / orcid.org/0000-00028885-1963

* Correspondencia: $\underline{\text { dr esau@hotmail.com }}$
}

Recibido: 3/7/21; Aceptado: 28/9/21; Publicado: 4/10/21

Resumen: Las tecnologías en información y comunicación (TICss) son herramientas que se utilizan para procesar, administrar y compartir la información mediante diversos soportes tecnológicos. La necesidad actual de que todo profesional cuente con los conocimientos básicos en el área de inglés, informáTICsa y gerencia, es también válida para la medicina. Nos propusimos analizar los factores asociados al uso de TICss en médicos residentes $(n=59)$ de Medicina Familiar (MF) de la Unidad de Medicina Familiar No. 24 del Instituto Mexicano del Seguro Social (IMSS); se aplicó el Instrumento Tecnología en Información y Comunicación en médicos residentes, publicado por Veloz MG y col. 2012. El 98.6\% disponen de computadora portátil, $98 \%$ disponen de internet en su domicilio, el $69.5 \%$ ha parTICsipado en cursos en línea y se encontró una asociación entre el grado académico y nivel de dominio de internet con una $\mathrm{p}=.013$, eoncontrándose que el $49 \%$ de los residentes tienen un dominio regular en la computadora. Concluimos que el dominio regular de la computadora en médicos residentes es bajo, aunque existe una asociación entre el grado académico y el nivel de dominio de internet. Las TICs están cada vez más incorporadas en los procesos de formación y actualización de médicos residentes. La falta de dominio del idioma inglés no dificulta el uso de internet y la brecha generacional en el uso de TICs se va cerrando poco a poco, siendo estas herramientas útiles en el campo clínico de nuestros residentes.

Palabras clave: tecnologías en información y comunicación, residentes de medicina familiar.

\begin{abstract}
Information and communication technologies (ICT) are tools used to process, manage and share information through various technological supports. The current need for every professional to have basic knowledge in the area of English, IT and management is also valid for medicine. We set out to analyze the factors associated with the use of ICT
\end{abstract}


in medical residents $(n=59)$ of Family Medicine $(F M)$ of the Family Medicine Unit No. 24 of the Mexican Institute of Social Security (IMSS); The Information and Communication Technology Instrument was applied to resident physicians, published by Veloz MG et al. 2012. $98.6 \%$ have a laptop, $98 \%$ have internet at home, $69.5 \%$ have parTICsipated in online courses and an association was found between academic degree and level of internet proficiency with $\mathrm{p}=.013$, finding that $49 \%$ of residents have a regular domain on the computer. We conclude that regular computer proficiency in resident physicians is low, although there is an association between academic degree and level of internet proficiency. ICTs are increasingly incorporated in the training and updating processes of resident doctors. The lack of command of the English language does not hinder the use of the internet and the generation gap in the use of ICT is closing little by little, these tools being useful in the clinical field of our residents.

Keywords: information and communication technologies, family medicine residents.

\section{Introducción}

Los currículos de la enseñanza de la medicina se relacionan con las necesidades sociales de cada momento histórico, de manera que el modelo médico se convierte en la "aspiración" o guía para su diseño y ejecución (1). En los últimos tiempos, se han introducide las tecnologías de la información y comunicación (TICs) para la expansión de los procesos formativos en el intercambio de información médica. Las TICs son herramientas que permiten el acceso a la información por medio de dispositivos electrónicos de fácil manejo que conducen a la adquisición de nuevos conocimientos. Esta incorporación de las TICs en el área Médica actualmente se encuentra en un proceso de transición. De ahí la importancia de conocer las limitantes de los profesionales de la salud y gestionar programas que derriben estas barreras.

Actualmente el papel de las TICs en la sociedad es muy importante porque ofrecen muchos servicios como correo electrónico, búsqueda de información, banca online, descarga de música y cine, comercio electrónico, etc. Por esta razón las TICs han incursionado fácilmente en diversos ámbitos de la vida, entre ellos, el de la educación (2). La utilización de las TICs ha impulsado de forma acelerada el desarrollo científico-tecnológico, surgiendo nuevos escenarios docentes y la utilización de nuevos medios de enseñanza, donde han determinado la aparición de nuevos roles para las instituciones educativas, los docentes y los estudiantes, así como en el desarrollo de materiales de apoyo al proceso de enseñanza-aprendizaje $(3,4)$. El mundo vive un proceso de transformación no planificada que afecta la forma en la cual aprendemos. Exige ciudadanos con habilidades y competencias que les permitan desempeñar de forma adecuada, habilidades y competencias que no solo deben desarrollar los futuros médicos solo como ciudadanos, sino también dentro de sus procesos de formación debido a las dinámicas particulares que se están generando en su campo disciplinar gracias a las nuevas tecnologías $(5,6)$. Los avances tecnológicos aparecen para dar respuesta a las necesidades de estar en continua conexión con la información, lo que va a configurar un nuevo paradigma social, cultural y educativo (7). Un factor clave de este cambio se sustenta en las nuevas TICs. En el área de la salud el crecimiento de internet como medio de comunicación masivo ha revolucionado el manejo e intercambio de información en medicina. La búsqueda automatizada, el acceso a literatura en formato electrónico 
y el intercambio de texto, imágenes y sonidos son algunas de las características que hacen de internet un elemento imprescindible, dentro de la práctica médica y de los actuales estándares de educación médica (8). La introducción de las TICs en el proceso de enseñanza-aprendizaje evoluciona constantemente, tiene gran repercusión en la expansión de procesos formativos que utilizan la modalidad a distancia y semipresencial, pues ofrece la posibilidad de aumento de la interacción entre el profesor o tutor y el estudiante, la posibilidad de acceso en el momento y lugar deseado y la adecuación a las características personales de los que se benefician del programa de formación (9). Las TICs constituyen una herramienta de indudable valía para la transformación socialmente positiva de dicho proceso (10).

Algunos estudios señalan la importancia de evidenciar las deficiencias en el entrenamiento de los residentes sobre TICs al inicio del posgrado, aunque es de esperarse que, generacionalmente, los nuevos residentes exhiban gradualmente mayores y mejores competencias en la utilización de las TICs de forma general. El proceso de formación básica y posgraduada, que conforman un todo continuo, estará acompañado de la modernización de los procesos de aprendizaje con la consecuente elevación de las competencias de los graduados para dar satisfacción a los problemas de salud que se le planteen (11). Las TICs son consideradas una herramienta que facilita los accesos a la información médica de forma muy cambiante, lo que obliga a las instituciones de salud a invertir en recursos (12). Las TICs en el área de la salud ha permitido que los médicos de atención primaria las adopten paulatinamente, pero aún persisten barreras para poder acceder a ellas y explotar todo el potencial que estas tienen (13). Una de las principales limitantes a las que se enfrentan los residentes es la falta de equipos de cómputo con acceso a internet para su uso académico, lo que facilitaría el acceso a artículos recientes, guías de práctica clínica que apoyen el abordaje y tratamiento de los pacientes, plataformas académicas, entre otros (14). Por todo ello, nos planteamos analizar cuáles son los factores que se asocian con el uso de las Tecnologías de la Información y Comunicación en residentes de medicina familiar de la Unidad de Medicina Familiar No. 24 del Instituto Mexicano del Seguro Social (IMSS), Tepic Nayarit México.

\section{Métodos}

\subsection{Diseño}

Se realizó un estudio descriptivo, correlacional y transversal, mediante la aplicación del instrumento Uso de Tecnologías en información y comunicación en médicos residentes, elaborado y validado por Veloz MG y colaboradores (3). Se elaboró una base de datos y se analizó con el programa SPSS ${ }^{\circledR}$ versión 26, obteniéndose la estadística descriptiva y para la correlación de variables la prueba de Chi cuadrado con una $\mathrm{p}<.05$ de nivel de significación.

\subsection{ParTICsipantes}

La población de estudio fue conformada por el 100\% de residentes de MF que cursaban algún grado en el ciclo académico 2017-2018 de la Unidad de Medicina Familiar no. 24 del Instituto Mexicano del Seguro Social, en Tepic Nayarit. Se 
incluyeron los residentes que aceptaron participar, de ambos sexos, sin importar la edad, ni universidad de procedencia. Todos firmaron la carta de consentimiento informado, y completaron el instrumento de forma voluntaria.

\subsection{Dimensiones exploradas}

- Tecnologías de la información y comunicación: se valoraron los tipos de TICs que dispone el residente de MF, el nivel de dominio de las TICs se valoró mediante una escala tipo Likert que variaba desde muy bajo hasta muy alto.

- Tiempo dedicado a actividades relacionadas con educación: en esta dimensión se valoró el tiempo promedio en horas que emplea cada día para actividades educativas en general, cuanto tiempo en promedio por día utiliza las diferentes TICs, y del total de tiempo que utiliza las TICs, cuanto tiempo considera que emplea en forma efectiva para actividades relacionadas con la educación.

- Uso de internet: en esta dimensión se cuestionó sobre los buscadores que utiliza para descargar información científica, si realiza otra actividad en forma simultánea con las actividades educativas, tales como ver videos, escuchar música, redes sociales, entre otras.

- Comprensión del idioma inglés: se valoró la percepción del nivel de comprensión, que tienen del idioma inglés en una lectura de cualquier tema, expresado en porcentaje, y de igual forma en una lectura de publicaciones médicas científicas, y si consideran que la falta del dominio del idioma inglés les dificulta la búsqueda de información médica científica, en una escala tipo Likert que variaba desde mucho a nada.

- Preferencias de educación tradicional y TICs: se valoró sobre la participación en blogs y/o foros de discusión en línea, si han llevado algún curso en línea, si han estado presentes en videoconferencias, y si no han realizado ninguna actividad relacionada con educación en línea, el motivo principal por lo que no lo han realizado, y finalmente su percepción de la educación tradicional y el uso de TICs.

\section{Resultados y Discusión.}

Se estudiaron un total de 59 residentes distribuidos de la siguiente manera, 33 hombres y 26 mujeres (Tabla 1), con un rango de edad de 32 años en promedio, mínimo de 24 años y máximo 43. En el grado académico, 29 de primero, 20 de segundo y 10 de tercer grado. En cuanto a la disponibilidad de TICs, 49 disponen de equipo de cómputo de escritorio, 57 cuentan con computadora portátil, 58 poseen Smartphone, 58 cuentan con internet en su domicilio, y sólo 48 disponen de internet en sus dispositivos móviles.

Sobre el nivel de dominio de la computadora, un $49 \%$ tiene un nivel regular, $39 \%$ alto y $20 \%$ muy alto. En el nivel de dominio internet, el $44 \%$ tienen un nivel regular, $36 \%$ alto y $20 \%$ muy alto. El nivel de dominio de redes sociales es alto con un $33.4 \%$, el tiempo empleado en uso de TICs es en promedio entre 2 y 4 horas (figura 1). Dentro de los buscadores más utilizados se encuentra Google académico en un $40 \%$ y el $59.3 \%$ de los residentes realizan otra actividad simultanea como uso de redes sociales, ver televisión o escuchar música. 


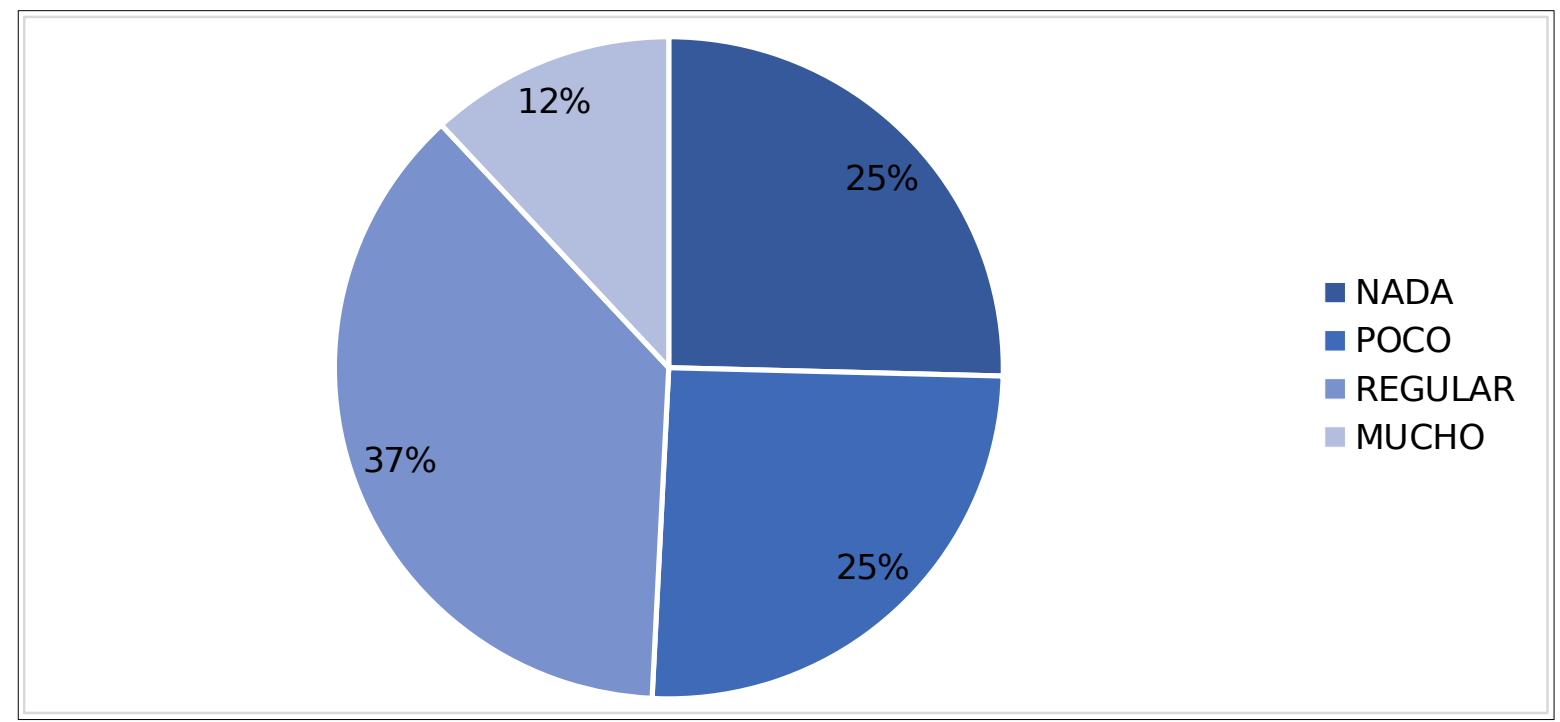

Figura 1. Tiempo empleado en el uso de TICs

En cuanto a la comprensión del idioma inglés en cualquier tema, el 52\% tiene un grado de comprensión entre un 60-85\%; en temas médicos, el $49 \%$ tiene un nivel del $60-85 \%$, y un $37 \%$ de los residentes indica que su bajo nivel de inglés hace que el uso de las TICs sea sólo regular (figura 2).

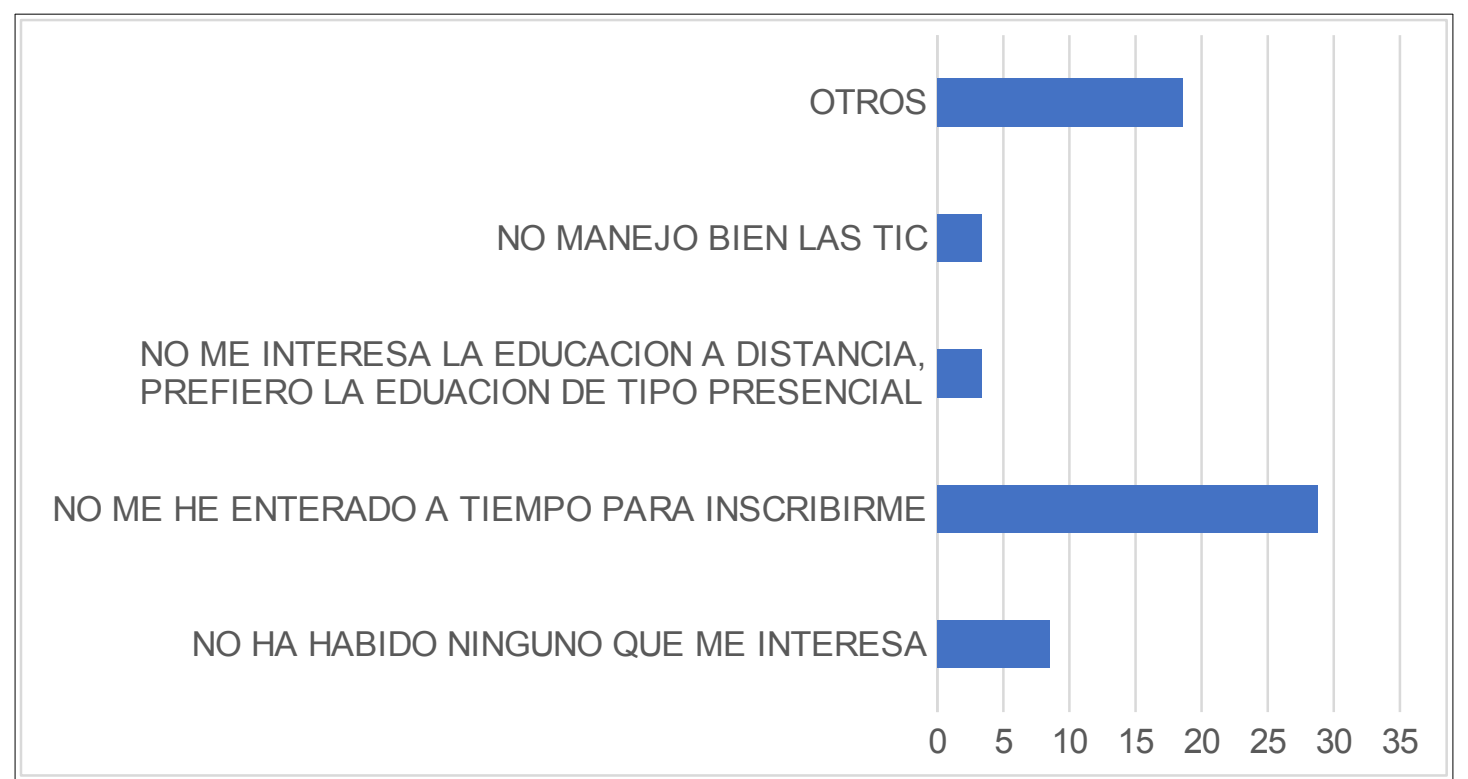

Figura 2. Dificultad en el uso de TICs por falta de dominio del idioma inglés.

El 54.2\% no ha participado en blogs, solamente el $69.5 \%$ ha realizado cursos en línea y el $61 \%$ no ha participado en videoconferencias. Los motivos por los que no han participado se muestran en la figura 3. El motivo principal es que no se han enterado a tiempo con un $28.8 \%$. Y de los que participaron $(n=46)$, a un $78 \%$ les gustó participar aunque al 5.1 les pareció aburrido; el 78\% considera que les ayudó a adquirir nuevos conocimientos, al 38\% les gustaría más actividades virtuales; finalmente, los tipos de curso que les gustaría recibir son de urgencias $(23.7 \%)$ y de medicina familiar, con un $17 \%$. 


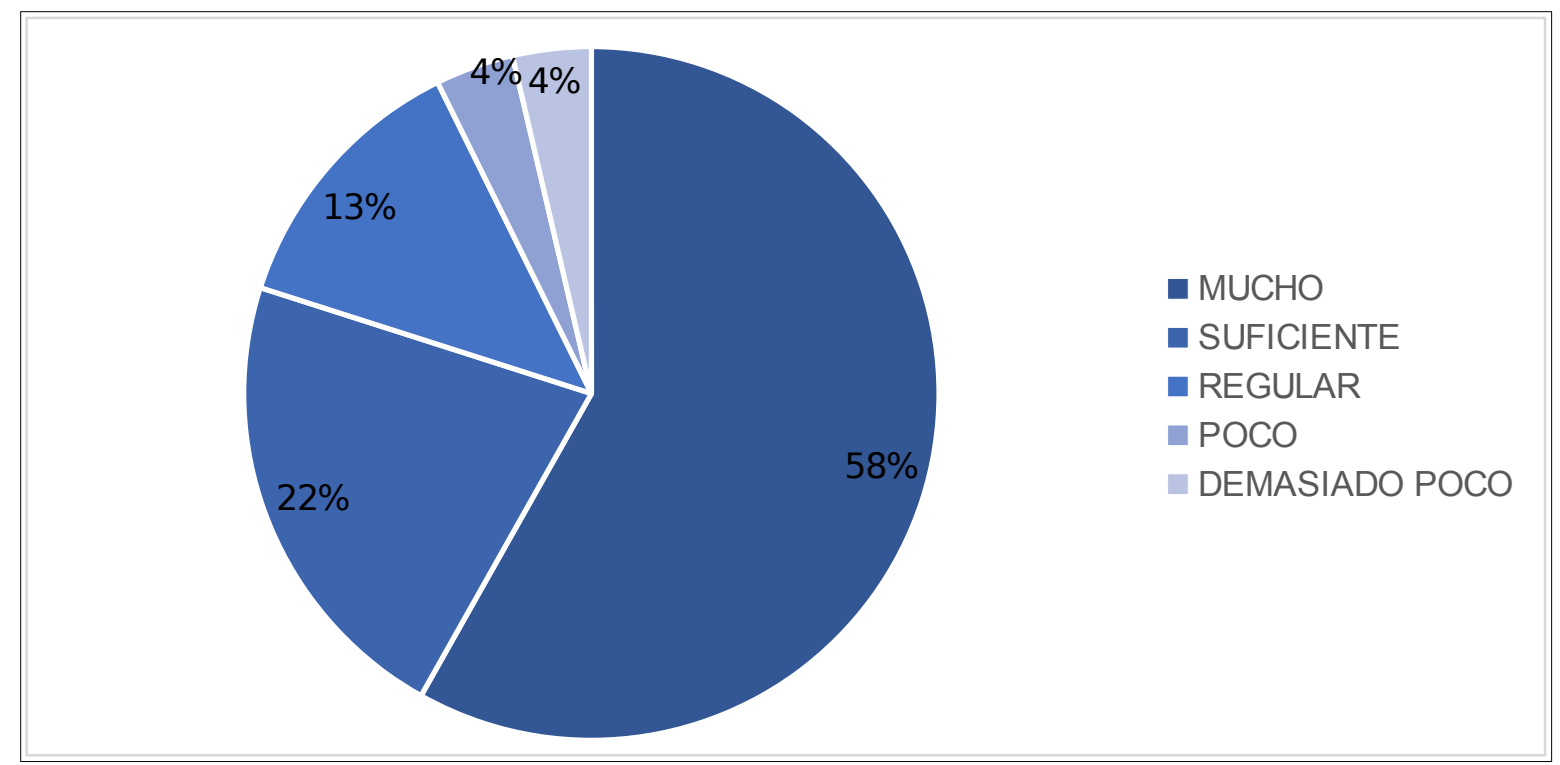

Figura 3. Motivos por los que no participan en TICs.

Dentro de las preferencias para profundizar en temas médicos en internet, el $54 \%$ lo usan mucho, mientras que en libros la preferencia es del $45.8 \%$, las videoconferencias las prefieren un $25.4 \%$ o de cursos en líneas un $22 \%$, exposición de temas por otra persona en vivo $(28.8 \%)$ y temas en línea en tiempo real $(25.4 \%)$. Se analizó la relación entre el grado académico y el nivel de dominio de internet a través de la prueba exacta de Fisher, encontrando que existe relación entre dichas variables $(p=.013)$ como lo reflejamos en la tabla 1 , donde se observa que los residentes de primer año tienen un nivel de dominio de internet muy alto.

De igual forma exploramos la relación del grado académico y el nivel de dominio de redes sociales encontrando nuevamente que los residentes de primer año tienen un nivel de dominio muy alto $(p=0.43)$. No se encontró relación entre el grado académico y la falta de dominio del idioma inglés en la dificultad para usar las TICs, $\mathrm{p}=0.8$ (tabla 2). Finalmente, no se encontró relación con la edad, grado académico para la participación en blogs o cursos en línea.

Tabla 1. Relación entre el grado académico y el nivel de dominio de Internet

\begin{tabular}{|c|c|c|c|c|c|}
\hline \multicolumn{2}{|c|}{} & \multicolumn{3}{|c|}{ Nivel dominio internet } & Total \\
\cline { 3 - 6 } \multicolumn{2}{|c|}{} & MUY ALTO & ALTO & REGULAR & \\
\hline \multirow{3}{*}{ Grado } & R1 & 10 & 10 & 9 & 29 \\
\cline { 2 - 6 } & R2 & 1 & 5 & 14 & 20 \\
\cline { 2 - 6 } & R3 & 1 & 6 & 3 & 10 \\
\hline \multicolumn{2}{|c|}{ Total } & 12 & 21 & 26 & 59 \\
\hline
\end{tabular}


Tabla 2. Relación entre el grado académico y la falta de dominio del idioma inglés en el uso de TICs.

\begin{tabular}{|c|c|c|c|c|c|c|}
\hline & \multicolumn{4}{|c|}{ Falta dominio inglés dificulta uso de TICs } & \multirow[t]{2}{*}{ Total } \\
\hline & & NADA & $\mathrm{POCO}$ & REGULAR & MUCHO & \\
\hline \multirow[t]{3}{*}{ Grado } & R1 & 9 & 8 & 10 & 2 & 29 \\
\hline & $\mathrm{R} 2$ & 4 & 4 & 8 & 4 & 20 \\
\hline & R3 & 2 & 3 & 4 & 1 & 10 \\
\hline \multicolumn{2}{|c|}{ Total } & 15 & 15 & 22 & 7 & 59 \\
\hline
\end{tabular}

Como se ha comentado, en el presente trabajo se encontró que las TICs ya son parte de la formación de residentes y su uso va superando la barrera generacional, siendo estas una herramienta útil en su proceso de aprendizaje, caracterísTICsa similar que se presentó en la formación de residentes médicos de la universidad veracruzana (14). En relación a la disponibilidad de TICs se encontró que solo el $83.1 \%$ cuentan con una computadora de escritorio, comparado con el 100\% que reportan Veloz y cols. (3) En su estudio, sin embargo, encontramos que $98.3 \%$ de nuestros residentes tienen acceso a internet en su domicilio comparado con el $86 \%$. El nivel de dominio del internet los resultados son similares, donde los residentes manifiestan un nivel de alto a muy alto. Es importante mencionar que, en nuestro estudio encontramos una brecha generacional, donde los residentes de primer año cuentan con un nivel de dominio mayor, que los residentes de grados superiores. Los residentes participantes pasan más tiempo en el uso de TICs en promedio 4 a 2 horas, en comparación con el reportado por Veloz y cols. (3) de 30 minutos a 8 horas. En nuestro estudio, aproximadamente la mitas de los residentes comprende adecuadamente la lectura de temas médicos en inglés mientras que solo el $25 \%$ de los residentes del estudio de Veloz y cols. (3) lo hacían. Es muy posible que esta nueva generación de residentes venga mejor preparada en el idioma inglés que los anteriores, ya que es bien conocido que la mayoría de la bibliografía de uso habitual está en este idioma.

\section{Conclusiones}

- El dominio regular de la computadora en médicos residentes es bajo.

- Existe asociación entre el grado académico y nivel de dominio de internet.

- Un poco más del $50 \%$ de los médicos residentes parTICsipa en blogs y cursos en línea.

- No existe relación en la falta de dominio del idioma inglés y el uso de internet.

- Las TICss se incorporan cad avez más en los procesos de formación como de actualización de los médicos residentes.

- La brecha generacional y el uso de las TICss se va cerrando poco a poco, siendo estas herramientas útiles en el campo clínico de nuestros residentes.

Financiación: No ha habido financiación

Declaración de conflicto of interés: Los autores declaran no tener ningún conflicto de intereses.

\section{Referencias}

1. Díaz ME, Martínez V, Ramos Ramírez MR, Romero Monteagudo ME. El currículo y el profesor en la transformación del binomio prácTICsa médica. Rev Cubana Med Gen Integr [Internet]. 2012 [cited 2017 Jul 26];299-308. Available from: http://scielo.sld.cu/scielo.php?script=sci arttext\&pid=S086421252012000300009\&lng=es

2. UNAM. Tutorial de estrategias de aprendizaje | TEA [Internet]. 2013 [cited 2017 Jul 26]. Available from: https://tutorial.cch.unam.mx/\#informacion 
3. Veloz-Martínez MG, Almanza-Velasco E, Uribe-Ravell JA, Libiend-Díaz González L, Quintana-Romero V, Alanís-López P. Uso de tecnologías en información y comunicación por médicos residentes de ginecología y obstetricia. Investigación en educación médica [Internet]. 2012 [cited 2017 Jul 24];1(4):183-9. Available from: http://www.scielo.org.mx/scielo.php?script=sci_arttext\&pid=S200750572012000400005\&lng=es\&nrm=iso\&tlng=es

4. Saiz G del C, Saiz GD del C, Gómez GS, Martínez MG. Tecnologías de la Información y las Comunicaciones: desafío que enfrenta la universidad de ciencias médicas. EDUMECENTRO [Internet]. 2018 Jan 1 [cited 2021 Jun 3];10(1):168-82. Available from: http://www.revedumecentro.sld.cu/index.php/edumc/arTICsle/view/908

5. Juanes Méndez JA. Current status of new technologies in the teaching of experimental sciences and medicine in parTICsular. Vol. 17, Educación Medica. 2016; 17: 1-2. https://doi.org/10.1016/j.edumed.2016.03.001

6. Boude FO. PediaTICs: development of competencies in ICT through projectbased learning. Revista
Cubana
de
Educación
Médica
Superior.
2011;25(2):116-124.

https://www.medigraphic.com/cgi-bin/new/resumenI.cgi?IDARTICsULO=31815

7. Briz-Ponce L, Juanes-Méndez JA, García-Peñalvo FJ. Dispositivos móviles y apps: CaracterísTICsas y uso actual en educación médica. NováTICsa. Revista de la Asociación de Técnicos en InformáTICsa. 2015;231: 86-91. $\quad$ https://repositorio.grial.eu/bitstream/grial/424/1/Art\%C3\%ADculo\%20NovaTICsa_espa \%C3\%B1ol.pdf

8. Carlos D, Quispe L. Maestros y computadoras portátiles en el Perú: ¿por qué no se usan las computadoras portátiles? [Internet]. Apertura. 2015 [cited 2017 Jul 26]. Available from: http://www.udgvirtual.udg.mx/apertura/index.php/apertura/arTICsle/view/585

9. Prieto Díaz V, Quiñones La rosa I, Ramírez Durán G, Fuentes Gil Z, Labrada Pavón T, Pérez Hechavarría $\mathrm{O}$, et al. Impacto de las tecnologías de la información y las comunicaciones en la educación y nuevos paradigmas del enfoque educativo. Educ Med Super [Internet]. 2011 [cited 2017 Jul 26];1:95-102. Available from: http://scielo.sld.cu/scielo.php?script=sci_abstract\&pid=S086421412011000100009\&lng=es\&nrm=iso

10. Candelaria BJC, Acosta CC, Ruíz PFR. Nefroweb: alternativa para la superación profesional de postgrado sobre Nefrogeriatría. Rev Ciencias Médicas [Internet]. 2017 [cited 2017 Sep 3];21:399-405. Available from: http://www.revcmpinar.sld.cu/index.php/publicaciones/arTICsle/view/2975

11. Peña E, Sandrea Jiménez MC, Avellán Rodríguez I. Intermed: una herramienta para la actualizacion en histologia e inmunologia nasal para residentes de otorrinolaringologia. Vitae [Internet]. 2013 [cited 2017 Jun 4];0(32). Available from: http:/ / saber.ucv.ve/ojs/index.php/rev_vit/arTICsle/view/4843\#.YLr4OM_r63M.mendeley

12. Culebro-Trujillo R, Contreras-Campos NE, Montiel-Jarquín ÁJ, Barragán-Hervella RG, López-Cázares G, García-Villaseñor A, et al. User Satisfaction on Computerised Information Sources in a Third Level Medical Facility. Educacion Medica. 2016 18(3):188-194. https://doi.org/10.1016/j.edumed.2016.06.017

13. Vázquez-Martínez VH, Ortega-Padrón M. Uso de las tecnologías de la información y la comunicación en médicos de atención primaria. Atención Familiar [Internet]. 2016 Feb 15 [cited 2017 Jun 4];23(1):19-23. Available from: https://www.medigraphic.com/cgi-bin/new/resumen.cgi? IDARTICsULO=62477\#.YLr7edtIaD4.mendeley

14. Olmedo Canchola VH, Andoney Mayén JV, Heinze Martin G, Olmedo Canchola VH, Andoney Mayén JV. Uso de las tecnologías de la información y comunicación (TICs) en las residencias médicas en México. Acta médica Grupo Ángeles [Internet]. 2017 [cited 2018 Jan 4];15(2):150-3. Available from: http:/ / www.scielo.org.mx/scielo.php?script=sci arttext\&pid=S1870$\underline{72032017000200150 \& \operatorname{lng}=\mathrm{es} \& n r m=\text { iso\&tlng=es }}$

(C) 2021 por los autores. Enviado para su publicación en acceso abierto bajo los términos y condiciones de la licencia Creative Commons Attribution (CC BY) (http://creativecommons.org/licenses/by/4.0/). 